\title{
Radiometers based on SIS mixers to measure SZ effect from galaxy clusters
}

\author{
M. Gervasi ${ }^{\text {a,* }}$, S. Banfi ${ }^{\text {a }}$, G. Boella ${ }^{\text {a }}$, A. Passerini ${ }^{\text {a }}$, V. Natale ${ }^{\text {b }}$, G. Sironi ${ }^{\text {a }}$, \\ A. Tartari ${ }^{\text {a }}$, M. Zannoni ${ }^{\text {a }}$ \\ a Department of Physics “G. Occhialini”, University of Milano Bicocca, Piazza della Scienza, 3, 20126 Milano, Italy \\ ${ }^{\mathrm{b}}$ IRA-INAF, Largo E. Fermi, 5, 50125 Firenze, Italy
}

Available online 23 January 2007

\begin{abstract}
We present a project to realize radiometers devoted to the observation of the Sunyaev-Zeldovich effect towards galaxy clusters. Radiometers are based on SIS mixers and are working at three frequency bands (around 94, 240 and $345 \mathrm{GHz}$ ) covering both the positive and negative part of the spectral displacement. The radiometers have been developed to be used at a telescope of 3 m class, like MITO. The observation program would be completed performing low frequency measurements first at the Noto radiotelescope (at $43 \mathrm{GHz}$ ) and then at SRT (at 40 and $90 \mathrm{GHz}$ ).
\end{abstract}

(C) 2006 Elsevier B.V. All rights reserved.

PACS: 98.80.-k; 95.55.- $\mathrm{n}$

Keywords: Cosmic microwave background; Sunyaev-Zeldovich effect; Radiometers; SIS mixers

\section{Contents}

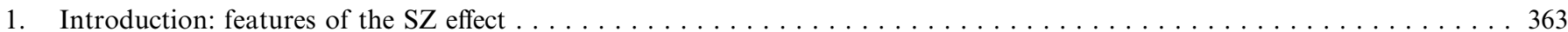

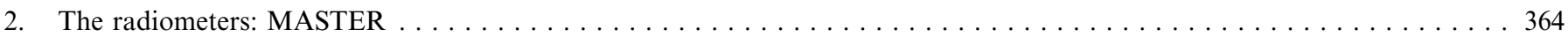

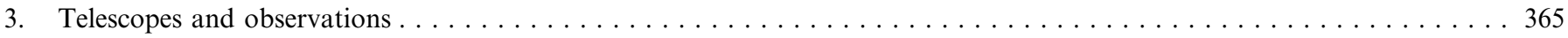

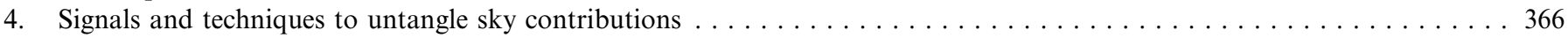

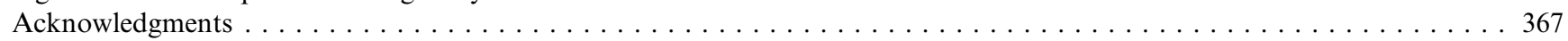

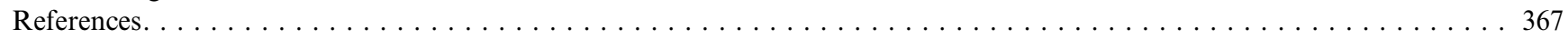

\section{Introduction: features of the SZ effect}

The Sunyaev-Zeldovich (SZ) effect, i.e. the comptonization of the CMB spectrum against a cluster of galaxies, has been observed till now by using different experimental approaches (for a review see Birkinshaw, 1999; Carlstrom et al., 2002). Single dish telescopes have been successfully

\footnotetext{
* Corresponding author.

E-mail address: massimo.gervasi@mib.infn.it (M. Gervasi).
}

used, as well as interferometers. Bolometric detectors are usually adopted at high frequency, while coherent receivers are actually used in the Rayleigh-Jeans region of the CMB spectrum. The full spectral characterization of the classical thermal SZ effect $\left(\Delta T_{\text {SZ-th }}\right)$ can be done by observing the signal in the frequency range from $90-100 \mathrm{GHz}$ upto $350-400 \mathrm{GHz}$. In this frequency range bolometers are largely more sensitive than coherent radiometers, because of both the larger bandwidth and the lower noise level. But a precise evaluation of the parameters is possible only after 
a precise spectral characterization of the SZ effect. The amount of the effect is well described by the comptonization parameter $y$, related to the optical depth $\left(\tau_{\mathrm{e}}\right)$ of the ionized intracluster medium (ICM). The frequency dependence $\left(x=h v / k T_{\mathrm{CMB}}\right)$ of $\Delta T_{\mathrm{SZ} \text {-th }}$ can be described by the following equations:

$$
\begin{aligned}
& \frac{\Delta T_{\mathrm{SZ}-\mathrm{th}}}{T_{\mathrm{CMB}}}=f_{0}(x) y ; \\
& f_{0}(x)=\left[x \frac{\mathrm{e}^{x}+1}{\mathrm{e}^{x}-1}-4\right] ; \\
& y=\int n_{\mathrm{e}} \frac{k T_{\mathrm{e}}}{m_{\mathrm{e}} c^{2}} \sigma_{\mathrm{T}} \mathrm{d} \ell .
\end{aligned}
$$

The bandwidth and the relative efficiency are not easily measured in bolometric devices. Such a characterization is much simpler in coherent systems, where the frequency band can be well evaluated and tuned. This is the main reason for using a coherent detector also in the high frequency region of the SZ spectrum. In particular it is important to make a spectral coverage around the cross-over frequency $x_{0}$, i.e. the frequency where the thermal SZ effect vanishes. Here the measurements are very sensitive also to: the kinetic SZ effect, which accounts for the peculiar motion (v) of the cluster in the CMB rest frame; the relativistic corrections to the thermal SZ, and other corrections.

The relativistic correction can be taken into account putting a frequency dependent additional term $\delta\left(x, T_{\mathrm{e}}\right)$ to $f_{0}(x)$ which becomes $f(x)$ (see Carlstrom et al., 2002; Itoh et al., 1998):

$f(x)=f_{0}(x)\left[1+\delta\left(x, T_{\mathrm{e}}\right)\right]$.

The correction term $\delta\left(x, T_{\mathrm{e}}\right)$ up to $k T_{\mathrm{e}} \leqslant 15 \mathrm{keV}$ can be written as a polynomial expansion of the parameter $\theta_{\mathrm{e}}=k T_{\mathrm{e}} / m_{\mathrm{e}} c^{2}$. The relativistic corrections to the classical thermal SZ effect are effective in the high frequency side of the spectrum, and not negligible when $k T_{\mathrm{e}} \geqslant 5 \mathrm{keV}$. On the other hand the kinetic SZ effect $\left(\Delta T_{\mathrm{SZ}-\mathrm{k}}\right)$ is much more smooth with the frequency and will contribute like an off-set term:

$\frac{\Delta T_{\mathrm{SZ}-\mathrm{k}}}{T_{\mathrm{CMB}}}=-\tau_{\mathrm{e}} \frac{v}{c}$,

with

$\tau_{\mathrm{e}}=\int n_{\mathrm{e}} \sigma_{\mathrm{T}} \mathrm{d} \ell$.

These contributions induce both a shift in the "observed" cross-over frequency $x_{0}$ and a distortion of the thermal SZ differential spectral shape. The undistorted value of the cross-over frequency is $v_{0} \simeq 218 \mathrm{GHz}\left(x_{0} \simeq 3.83\right)$. A second order corrected cross-over frequency is written as (Itoh et al., 1998):

$x_{0} \simeq 3.830\left[1+1.1674 \theta_{\mathrm{e}}-0.8533 \theta_{\mathrm{e}}^{2}\right]$.

In order to untangle all these contributions a wide coverage of the SZ spectrum is necessary. Additional measurements at lower frequency, where the relativistic corrections are not effective, are also useful.
A multi-frequency approach is therefore mandatory, also in order to separate the signal coming from the local foregrounds and from the CMB anisotropies. Among the foregrounds we must consider the thermal dust emission of the cluster, whose emissivity scales as $v^{\alpha}(\alpha \sim 1.5)$. Also the anisotropic dust emission of our galaxy has to be also considered as a contaminant. Besides, radio sources contribute with a frequency power law scaling as $v^{-\beta}(\beta \sim 2-3)$. Finally the CMB anisotropies contribute to the observed signal, but the amplitude is related only to the angular scale, but not to the frequency (if considered in terms of CMB temperature), and it is not distinguishable from $\Delta T_{\mathrm{SZ}-\mathrm{k}}$ in the nonrelativistic limit.

\section{The radiometers: MASTER}

MASTER (Millimeter And Sub-millimeter Triple hEterodyne Receiver) is a system of three heterodyne receivers based on $\mathrm{Nb} / \mathrm{Al}-\mathrm{AlO}_{x} / \mathrm{Nb}$ SIS quasiparticle mixers to be placed in the focus of the MITO telescope (De Petris et al., 1996, 1999). The operating frequencies are 94, 240 and $345 \mathrm{GHz}$. The $345 \mathrm{GHz}$ mixer has been designed and manufactured by the KOSMA group at the University of Köln, while the $240 \mathrm{GHz}$ has been provided by the Department of Astronomy at UMASS. The $94 \mathrm{GHz}$ unit is not yet available, but prototypes have been developed at the I.N.Ri.M. in Torino and tested using the cryogenic test facility at the Physics Department of the University of Milano-Bicocca. The tunable RF bandwidth $(\Delta v)$ is around $10 \%$ of the central frequency $\left(v_{\mathrm{c}}\right)$ at $v_{\mathrm{c}}=94 \mathrm{GHz}(\Delta v=88$ $100 \mathrm{GHz})$ and at $v_{\mathrm{c}}=345 \mathrm{GHz}(\Delta v=330-370 \mathrm{GHz})$, while it is almost $0.25 v_{\mathrm{c}}$ at $v_{\mathrm{c}}=240 \mathrm{GHz}(\Delta v=210-270 \mathrm{GHz})$. The IF instantaneous bandwidth is $\delta v=600 \mathrm{MHz}$ at 94 and $345 \mathrm{GHz}$, while $\delta v=4 \mathrm{GHz}$ for the $240 \mathrm{GHz}$ unit.

The three RF front-ends will be assembled in a cryostat built around a pulse tube cooler, which can support a thermal input $\lesssim 700 \mathrm{~mW}$ at $\simeq 4 \mathrm{~K}$. Therefore both the mixers and the first low noise amplifiers can be accomodated on the cold stage. The front-end can provide an amplification around $30 \mathrm{~dB}$ just after the SIS. Once the signal in taken outside the cryostat through a coaxial $50 \Omega$ cable, it will be amplified again around $70-80 \mathrm{~dB}$ in the back-end by amplifiers operating at room temperature, before entering the crystal detector and the ADC converter. The study of the optical configuration is in progress, but the basic requirement to be fulfilled is to achieve the chance of a multi-frequency detection with the three channels working together simultaneously. Using just one window in order to minimize the thermal load, we have to make provision for: (1) a quasi-optical beam guiding system, in order to image the beam-waist of the feed horns into the Cassegrain focus with the desired magnification and (2) a couple of diplexers to split the radiation in a frequency dependent way. Both ellipsoidal mirrors and filters will be anchored at the $4 \mathrm{~K}$ stage in order to reduce the thermal noise.

MASTER will work as a triple Dicke-receiver, with the modulation of the signal taking place at the level of the 
focal plane (using a chopping wheel as reference) or, alternatively, by the sky-wobbling technique at constant elevation, which is the optimal choice to get rid of instrumental and atmospheric offsets.

Finally we point out as one on the IF chains can be used to feed an Acusto Optical Spectrometer provided by the Arcetri Astrophysical Observatory. This spectrometer has thousand spectral elements, covering a bandwidth of around $1 \mathrm{GHz}$ with a spectral resolution of around $1 \mathrm{MHz}$.

\section{Telescopes and observations}

A good scientific opportunity provided by a receiver with the properties we have just summarized, is that we can explore the thermal SZ effect around the crossover frequency with the most sensitive channel, while a simultaneous atmospheric monitoring can be done at $345 \mathrm{GHz}$ (see Fig. 1). In the same time, we expect a SZ detection where the effect is stronger, i.e. in the lowest frequency band. Moreover, we can exploit the different frequency channels in order to single out the different foregrounds with the typical power laws recalled in Section 1.

Concerning the foregrounds, an important remarks is that they exhibit a negative power-law scaling also in the spatial frequency domain (in the $\ell$ space, if we assume that the signal has been represented upon the $\left\{Y_{\operatorname{lm}}\right\}$ basis), as it happens in the case of the galactic dust (Masi et al., 2001). This fact suggests that the beam-throw should be kept wide enough in order to reach a reference (OFF) position well outside the cluster's atmosphere, but, conversely, the amplitude of the modulation is constrained on the opposite side by angular distribution of the foregrounds. From this point of view, with its three-field modulation system (Mainella et al., 1996), MITO is a flexible instrument suitable for the observation of sources with extent varying from few to tens of arcminutes (e.g. the extent of the COMA cluster).

While we are developing our triple system, we are planning and carrying on observational campaign devoted to the study of the Sunyaev-Zeldovich effect with existing coherent receivers. There are mainly two kinds of reasons driving this activity. First of all we can approach the technical difficulties hidden in continuum observations of weak signals with narrow bandwidth systems. As a second issue, we can observe clusters of galaxies in a frequency domain where different foregrounds overwhelm the faint cosmological signal. This is particularly useful if the same object is observed for example at MITO and at the forthcoming SRT (IRA 371/05, 2005). In fact we have shown elsewhere (Gervasi et al., 2006) that adding a low frequency channel we improve considerably the quality of the detection.

In the light of this aims, we have proposed to use the dual-polarization $43 \mathrm{GHz}$ receiver at the focus of the $32-$ $m$ VLBI antenna at Noto for a cosmological campaign. At this frequency, this telescope has a FWHM $\cong 55^{\prime \prime}$, comparable with the resolution achievable at MITO at $345 \mathrm{GHz}$. In order to check the performances of the telescope for this kind of astrophysics, we proposed to observe A773 as main target, since its SZ signature has been observed both with single dish telescopes and with interferometers, and therefore it is suitable for tests. Among the published detection we can mention that one (Tsuboi et al., 2004) at the 45-m Nobeyama telescope with a sixbeam focal-plane array based on SIS operating at $43 \mathrm{GHz}$, with $500 \mathrm{MHz}$ integration bandwidth. Besides A773 is an intermediate distancecluster $(z=0.22)$ and its core radius fits the beam of the Noto telescope so that no dilution effect is expected. Observations has been scheduled in January 2006, but a technical problem forced us to postpone the campaign at the beginning of May, when the

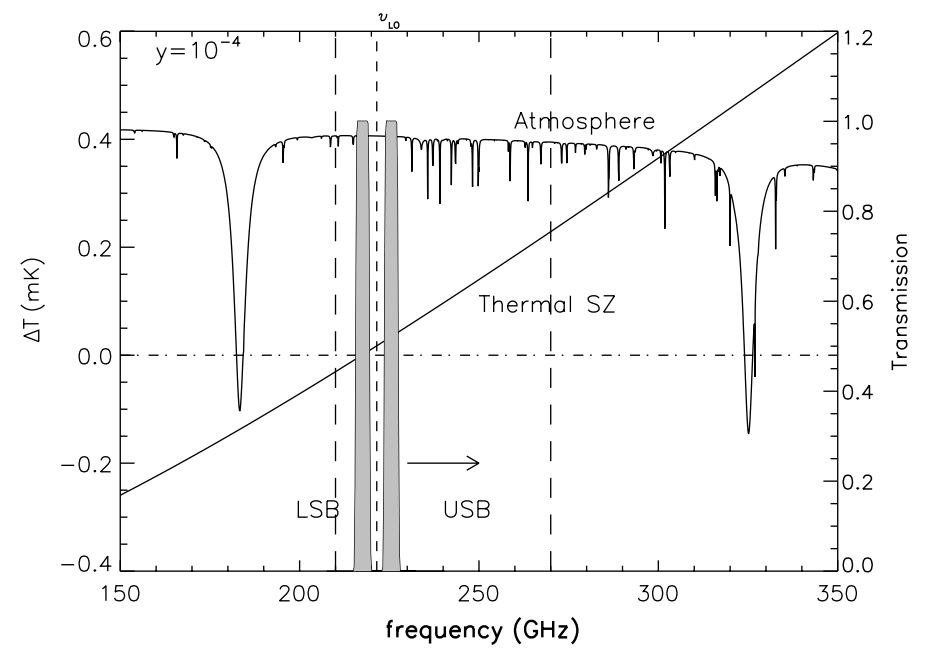

Fig. 1. The thermal SZ effect for a cluster with comptonization parameter $y=10^{-4}$ is shown in the frequency band around $240 \mathrm{GHz}$ (left scale). In the same plot the transmission window of the atmosphere at MITO is also represented (right scale) and, on the same scale, we show also upper and lower sideband (USB and LSB) of the SIS receiver. One of these two bands could be rejected in order to have the desired spectral purity (single side-band configuration). The dash-dotted line represents the null signal. The vertical dashed line singles out the tuning frequency of the local oscillator. The longdashed lines show the limits of the tuning bandwidth. 
atmospheric conditions are worse than those typical of winter-time. We observed A773 mainly during day-time, since it was always $\sim 90^{\circ}$ away from the Sun, while we observed A2163 during the night-time. The observing techniques will be shortly described in Section 4 .

This kind of observations carried on with the 32-m Noto antenna has the further aim of suggesting possible strategies to be implemented in the future at SRT, whose 40 and $90 \mathrm{GHz}$ channels could be suitable for the study of clusters of galaxies.

\section{Signals and techniques to untangle sky contributions}

The signal collected at the telescope is made of several contributions which can be of local origin, i.e. the ground and the atmospheric emission or celestial origin, i.e. sources and diffuse unwanted foregrounds. For our observations the possible contaminants are diffuse galactic synchrotron radiation and free-free emission (from regions close to the galactic disc) at the Noto $32-\mathrm{m}$ antenna in the $43 \mathrm{GHz}$ channel, and mainly galactic dust at $\mathrm{mm}$ and sub-mm wavelengths at the MITO telescope. Moreover, we cannot exclude a priori the presence of dust in the ICM, and also CMB primary anisotropies have to considered among the contaminants.

At the level of local signals, the atmosphere is the main obstacle able to hamper a fruitful cosmological campaign. In an oversimplified picture, it can be considered as a medium attenuating the sky signal by a factor $\exp [-\tau(h)](\tau$ is the optical depth, while $h$ is the zenith angle) and adding an amount of noise temperature $T_{\mathrm{n}, \mathrm{atm}}=T_{\text {atm }}[1-$ $\exp (-\tau(h))]$ proportional to the atmospheric temperature.
This suggests that it should be possible to get rid of this extra-noise subtracting the signals collected along two different lines of sight but with the same air-mass, obtaining, at the first order, the exact offset cancellation. But how exact is it? It is exact as far as the noise spectra of atmospheric fluctuations are white. In general, there are correlated fluctuations of relevant quantities, such as the temperature and the concentration of water vapor which is an absorbing/emitting component of the atmosphere at sub-mm wavelengths due to the onset of turbulent motions (Lena et al., 1998).

Atmospheric turbulence has rather complicated statistical properties which can be partly characterized with twopoint correlation functions both in space and time. In the simplest case, when a Kolmogorov spectrum is assumed for spatial perturbations, the 3D power spectral density of temperature fluctuations scales as $k^{-11 / 3}, k$ being the perturbation wavenumber, even if observations seems to suggest slightly different scaling laws (Beyer et al., 2003). At the same time, temporal power spectra exhibit a power-law of the kind $f^{-\beta}$, with $\beta=5 / 3$ preferred by the Kolmogorov theory, with the further assumption that fluctuations are frozen in the atmosphere and blown by the wind at constant velocity (Taylor hypothesis). Excess noise with respect to white noise floor has been observed from $0.1 \mathrm{~Hz}$ up to frequencies higher that $10 \mathrm{~Hz}$, while the external scale of turbulence can vary from few meters to hundreds of meters at altitudes varying from $10^{2} \mathrm{~m}$ to $10^{4} \mathrm{~m}$, even if turbulent motions are mainly concentrated in the low atmosphere (Travouillon et al., 2003; Short et al., 2003). In turn, the projection of the correlation length scales on the celestial sphere gives the maximum angular extent of the corresponding angular correlation scale.

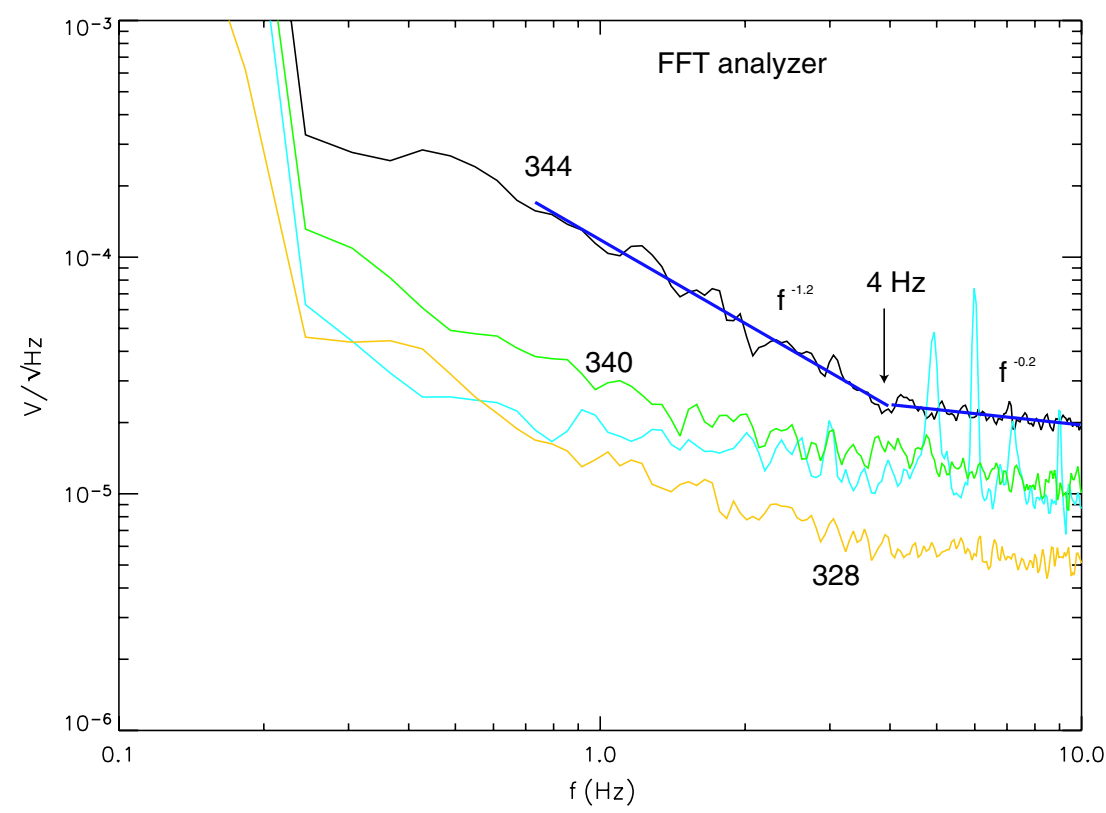

Fig. 2. The noise spectra of the receiver in total power configuration, obtained with the FFT analyzer, with the local oscillator pumping at different frequencies and with different power levels are reported. The two reported spectra at $340 \mathrm{GHz}$ have been measured looking through the telescope first at the closed randome (green) and then at the sky (light blue) while chopping at $6 \mathrm{~Hz}$. The noise floor, as expected, is lower in the second case, while the power at the wobbling frequency is evident. (For interpretation of the references in color in this figure legend, the reader is referred to the web version of this article.) 
The problem of the sky noise is unavoidable, especially in view of lasting cosmological campaigns, and can be faced with a careful site testing devoted to the reconstruction of noise spectra (see for example the overall noise spectrum obtained at MITO with the $345 \mathrm{GHz}$ SIS receiver, Fig. 2). This can be done at the hardware level at MITO, where a Dicke configuration allows to modulate the sky signal choosing properly the frequency and the amplitude of the oscillations of the secondary mirror. In this case the observer has access to the demodulated signal and can check in a straightforward way how the noise level moves changing the setting of some instrumental parameters. Moreover, if observations at several frequencies are planned, as in the case of MASTER, one could exploit the study of the correlation among different frequency channels to single out atmospheric fluctuations (Melchiorri et al., 1996). In fact this technique has already been successfully tested at MITO to obtain a multifrequency detection of the SZ effect in the Coma cluster of galaxies (De Petris et al., 2002). It's also important to recall that, as we have seen in the previous section, the amplitude of the beamthrow is constrained also by: (1) the angular size of the cluster and (2) by the angular power spectrum of the foregrounds.

At the 32-m antenna at Noto the observational problems are radically different. Due to the dimensions of the instrument, the beam switching is more critical, and in any case the modulation frequency is definitely lower than $1 \mathrm{~Hz}$ (so far beam switching at $0.25 \mathrm{~Hz}$ with $1^{\prime}$ beam-throw has been tested with the $43 \mathrm{GHz}$ receiver at the focus of the telescope). Moreover, unlike what happens at MITO, the system operates in a total power configuration, and demodulation has to be performed a posteriori at software level. When beam-switching has been adopted as observational technique, we decided also to observe two blank fields displaced $10^{\prime}$ in declination from the nominal position of the source, in order to single out the presence of signal offsets.

Another interesting chance that during the first SZ campaign has been investigated is the position switch moving the antenna at constant elevation, with the secondary mirror always positioned on-axis. In this case we have modu- lated the signal at $0.1 \mathrm{~Hz}$, with a beam-throw of $5^{\prime}$. The preliminary results obtained with this technique suggest that the scaling of the variance against the on-source integration time is steeper for night-time data than for daytime data. This means that, after the demodulation, we cannot observe a knee in the Allan-plot even considering a data-stream of several hours. These results are suggesting that we are effectively reducing the impact of atmospheric fluctuations. However, at the present status of the analysis we are not able to quote the ultimate level of sensitivity.

\section{Acknowledgments}

This activity is currently supported by MIUR through the PRIN-04 N.2004027755, Gemini-SZ program. We want to remember as Francesco Melchiorri was the proponent and the former scientific coordinator of this program. Authors acknowledge M. De Petris, L. Lamagna and S. De Gregori for their continuous support during the first test campaigns at the MITO telescope, and P. Leto for providing the observing schedules for the Noto telescope.

\section{References}

Beyer, J.T. et al., 2003. Appl. Opt. 42 (6), 908-921.

Birkinshaw, M., 1999. Phys. Rep. 310, 97-195.

Carlstrom, J.E., Holder, G.P., Reese, E.D., 2002. ARA\&A 40, 643-680.

De Petris, M. et al., 1996. New Ast. 1, 121-132.

De Petris, M. et al., 1999. New. Ast. Rev. 43, 297-315.

De Petris, M. et al., 2002. ApJ Lett. 574, L119-L122.

Gervasi, M. et al., 2006. Mem.S.A.It. Suppl. 10, 175-179.

IRA 371/05, IRA Internal Report, The Sardinia Radio Telescope (SRT)

Science and Technical Requirements, Report of the working group, 2005.

Itoh, N., Kohyama, Y., Nozawa, S., 1998. ApJ 502, 7-15.

Lena, P., Lebrun, F., Mignard, F., 1998. Observational Astrophysics. Springer-Verlag.

Mainella, G. et al., 1996. Appl. Opt. 35, 2246-2252.

Masi, S. et al., 2001. ApJ 553, L93-L96.

Melchiorri, B. et al., 1996. ApJ 471, 52-63.

Short, N., Fitelson, W., Townes, C.H., 2003. ApJ 599, 1469-1477.

Travouillon, T. et al., 2003. A\&A 400, 1163-1172.

Tsuboi, M. et al., 2004. PASJ 56, 711-721. 